\title{
Erratum
}

\section{Synthesis of Urea and Carbamate Glycosides Employing Unprotected Carbohydrates}

Yoshiyasu Ichikawa,* Shohei Kusaba, Takahiro Minami, Yumiko Tomita, Keiji Nakano, Hiyoshizo Kotsuki Synlett 2011, 1462.

The titles of the last columns in Tables 1 and 2 have accidentally shown the anomeric ratios as $\alpha / \beta$. In fact, they should read $\beta / \alpha$ instead.

In addition, on page 1462, left column, last paragraph, line 4 from the bottom, monomethylureid was misspelled as monomethylurid.

We apologize for these mistakes. 